\title{
Calorimetric indications of a cooperativity onset in the crossover region of dynamic glass transition for benzoin isobutylether
}

S. Kahle, K. Schröter, E. Hempel, and E. Donth

Citation: The Journal of Chemical Physics 111, 6462 (1999); doi: 10.1063/1.479942

View online: https://doi.org/10.1063/1.479942

View Table of Contents: http://aip.scitation.org/toc/jcp/111/14

Published by the American Institute of Physics

\section{Articles you may be interested in}

Dynamics of glass-forming liquids. III. Comparing the dielectric $\alpha$ - and $\beta$-relaxation of 1-propanol and $o$-terphenyl The Journal of Chemical Physics 107, 1086 (1997); 10.1063/1.474456

Dynamics of glass-forming liquids. V. On the link between molecular dynamics and configurational entropy The Journal of Chemical Physics 108, 9016 (1998); 10.1063/1.476348

Classification of secondary relaxation in glass-formers based on dynamic properties

The Journal of Chemical Physics 120, 857 (2004); 10.1063/1.1630295

Viscosity and shear response at the dynamic glass transition of glycerol The Journal of Chemical Physics 113, 9101 (2000); 10.1063/1.1319616

Nonexponential relaxations in strong and fragile glass formers

The Journal of Chemical Physics 99, 4201 (1993); 10.1063/1.466117

\section{PHYSICS TODAY}

WHITEPAPERS
ADVANCED LIGHT CURE ADHESIVES

READ NOW

Take a closer look at what these environmentally friendly adhesive systems can do
PRESENTED BY

8) MASTERBOND" 


\title{
Calorimetric indications of a cooperativity onset in the crossover region of dynamic glass transition for benzoin isobutylether
}

\author{
S. Kahle \\ Fachbereich Physik, Universität Halle, D-06099 Halle (Saale), Germany \\ and Institut für Festkörperforschung, Forschungszentrum Jülich, D-52425 Jülich, Germany \\ K. Schröter, E. Hempel, and E. Donth \\ Fachbereich Physik, Universität Halle, D-06099 Halle (Saale), Germany
}

(Received 2 October 1998; accepted 16 July 1999)

\begin{abstract}
Heat capacity spectroscopy (HCS), dielectric spectroscopy, and shear viscosity data are reported for liquid benzoin isobutylether (BIBE). Dielectric and viscosity peculiarities indicate the crossover region of dynamic glass transition at $T=(-8 \pm 8){ }^{\circ} \mathrm{C}$ where the extrapolated Johari Goldstein $\beta$ relaxation intersects the main transition trace in an Arrhenius plot. Although HCS does not reach the crossover frequency of order $\omega=2 \times 10^{6} \mathrm{rad} / \mathrm{s}$, the linear decrease of the square root of cooperativity as calculated from HCS data at lower frequencies indicates a cooperativity onset in the crossover temperature-frequency range. As BIBE belongs to another dielectric crossover scenario as the substances where such an onset could previously be observed, it seems that the cooperativity onset is a general property of the crossover region. (C) 1999 American Institute of Physics. [S0021-9606(99)50938-0]
\end{abstract}

\section{INTRODUCTION}

Dynamic heterogeneity of dynamic glass transition is an active field of experimental and theoretical research. The field is well reviewed by Sillescu, ${ }^{1}$ recent general reviews of the glass transition are Refs. 2-5. An important property of dynamic heterogeneity is its characteristic length $\xi_{\alpha}$. This length ${ }^{6}$ can be identified with the average size of cooperatively rearranging regions (CRR) introduced by Adam and Gibbs. ${ }^{7}$ A CRR is defined as a subsystem which, upon a sufficient thermodynamic fluctuation, can rearrange into another configuration independently of its environment.

This CRR definition by statistical independence leads directly ${ }^{8,9}$ to a calorimetric fluctuation formula for the average CRR size:

$$
N_{\alpha}=R T^{2} \Delta\left(1 / c_{\mathrm{v}}\right) / M \delta T^{2} \approx R T^{2} \Delta c_{p} / \bar{c}_{p}^{2} M \delta T^{2},
$$

where all variables on the right hand side can be determined from heat capacity spectroscopy (HCS). ${ }^{9}$ In Eq. (1), $N_{\alpha}$ is the cooperativity, defined as the volume of a CRR, $V_{\alpha}=\xi_{\alpha}^{3}$, reduced by the volume of one molecule with molecular mass $M, R$ the molar gas constant, $\Delta\left(1 / c_{\mathrm{v}}\right)$ the step height of the reciprocal specific heat at constant volume (approximated by $\Delta c_{p} / \bar{c}_{p}^{2}$ in this work), and $\delta T^{2}$ the $m s$ temperature fluctuation related to the dynamic glass transition of one CRR. ${ }^{8,9}$ In this paper, $\delta T$ is determined as the dispersion of a Gaussian fit for the imaginary part of isochronous dynamic heat capacity, ${ }^{10}$

$$
c_{p}^{\prime \prime}(T) \sim \exp \left[\left(T-T_{\max }\right)^{2} / 2 \delta T^{2}\right] .
$$

The proof, for Gaussian distributions in the framework of linear response, that this dispersion $\delta T$ is identical to the temperature fluctuation of a CRR will be published elsewhere. ${ }^{11}$ The typical characteristic length is $\xi_{\alpha} \approx 2 \cdots 3$ $\mathrm{nm}^{6}$ at the glass temperature, $T_{g}$, for substances where $T_{g}$ is far below the crossover temperature. This length corresponds to $N_{\alpha}$ of order 100 .

The crossover region of dynamic glass transition was first observed in polymers ${ }^{12}$ and later, by Johari and Goldstein, ${ }^{13}$ for many small-molecule glass formers. The crossover is indicated by an approach of the trace for the $\alpha$ transition-the conventional dynamic glass transition-to that of a typical activated Arrhenius process, $\beta$, in the Arrhenius plot. The latter was later called Johari and Goldstein process. It was Williams in $1966^{14}$ who first stated that the high-temperature process above the crossover should be considered as a distinct and separate process. In our paper this process is called $a$, the high-temperature process.

The crossover is further indicated by a peculiarity in the temperature dependence of viscosity. ${ }^{15,16}$ Usually, the $a$ and the $\alpha$ processes can reasonably be approximated by their own VFT functions for the viscosity. They have different parameters, especially different Vogel temperatures $T_{\infty}$ with, as a rule, $T_{\infty}(a)>T_{\infty}(\alpha)$. This behavior would be expected if $\alpha$ has a larger length scale than $a .^{17}$ It was confirmed by Stickel et al. ${ }^{18}$ for dielectric traces in the Arrhenius diagram, modeled by corresponding $\mathrm{WLF}^{19}$ functions for $a$ and $\alpha$ touching one another in the crossover,

$$
\log \omega_{\max }=\log \Omega-B /\left(T-T_{\infty}\right) .
$$

In Eq. (3), $\omega_{\max }$ is the frequency of the dielectric loss maximum at the temperature $T$. There are many other peculiarities in the crossover region, ${ }^{20,21}$ recent reviews are Refs. 22. Furthermore, the calorimetric contour map $c_{p}^{\prime \prime}(\log \omega, T)$ from HCS has a saddle between $a$ and $\alpha$ process at the crossover. $^{23}$

Different dielectric scenarios for the crossover region of dynamic glass transition were discussed so far. ${ }^{24,25}$ The two 


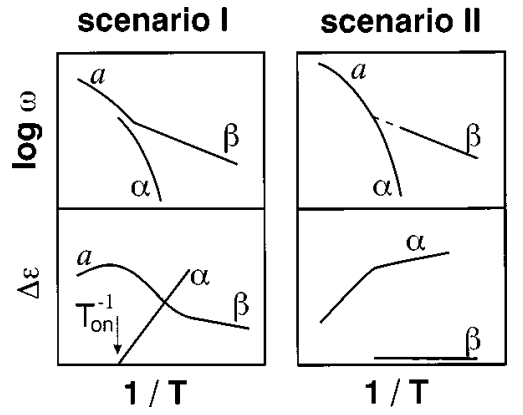

FIG. 1. Two schematic dielectric crossover scenarios. Top: Arrhenius diagram; bottom: intensities. The $\alpha$ onset temperature is indicated by $T_{\text {on }}$ in the I scenario. Details see text.

scenarios I and II in Fig. 1 are a generalization of the four scenarios (A,B,C,D) in Ref. 24: A,B $\rightarrow \mathrm{I}$; $\mathrm{C}, \mathrm{D} \rightarrow \mathrm{II}$.

Scenario I, as a rule, is observed if the dielectric relaxation strengths of $\beta$ and $\alpha$ relaxation are of the same order of magnitude, $\Delta \varepsilon_{\beta} \sim \Delta \varepsilon_{\alpha}$. It has a continuous $\beta a$ trace and $\beta a$ intensity, but the $\alpha$ intensity tends linearly to zero ${ }^{24,26-28}$ and has a separate $\alpha$ onset. Scenario II is observed for several small-molecule glass formers with a $\Delta \varepsilon_{\beta} / \Delta \varepsilon_{\alpha} \ll 1$ ratio. It is characterized by a continuous $\alpha a$ trace, the $\beta$ intensity is small, and the $\alpha a$ intensity is continuous with a bend in the crossover region. ${ }^{29-31}$

In all substances with the I scenario investigated so far, where the crossover is accessible (or nearly accessible) by HCS, we observed for $T<T_{\text {on }}{ }^{25}$

$$
\Delta c_{p} \sim\left(T_{\mathrm{on}}-T\right)
$$

and

$$
N_{\alpha}^{1 / 2} \sim\left(T_{\text {on }}-T\right) .
$$

Equation (5) means that the dielectric $\alpha$ onset is accompanied by a cooperativity onset in the I scenario. The question is if a cooperativity onset also exists for the II scenario.

The main experimental problem is that the crossover region for II scenario substances is usually in the mega-togigahertz frequency region, at present not accessible by HCS. We observed that at present benzoin isobutylether (BIBE) is the most promising II candidate for an HCS investigation. Its crossover frequency is at about $2 \times 10^{6} \mathrm{rad} / \mathrm{s}$, only two frequency decades above the upper frequency limit of our present HCS device developed to detect small and wide $c_{p}^{\prime \prime}(T)$ peaks. ${ }^{23,25,32}$

This frequency gap, however, generates specific problems when the cooperativity onset behavior should be detected with a certain confidence. In a way, they correspond to problems of critical-behavior studies in the early 1960's. The low experimental precision at that time did not allow, for one property, to determine $T_{c}$ and a critical exponent $x$ independently. Instead, only a function $x\left(T_{c}\right)$ could be determined. Other activities were needed, e.g., to fix $T_{c}$ first. Then the $x$ exponent could be determined from the function.

The application of such methods to our gap problem is supported by the experience ${ }^{10,25}$ that the onset behavior is well established even several decades (or several ten kelvins, respectively) below the onset itself. This is an advantage when compared to the critical state where the singularity is restricted to the order 1 kelvin around $T_{c}$.

In our paper we therefore combine many methods to find consistently an onset temperature $T_{\text {on }}$, an onset frequency $\omega_{\text {on }}$, and a cooperativity exponent $\nu_{g}$ from the ansatz

$$
N_{\alpha}^{\nu_{g}} \sim\left(T_{\text {on }}-T\right), \quad T<T_{\text {on }} .
$$

First we determine crossover temperatures and frequencies by the methods (i) to (iv) described in the following. They were then identified with the $\alpha$ cooperativity onset temperature $T_{\text {on }}$ and frequency $\omega_{\text {on }}$ from method (v) according to Eq. (6). A complex strategy is needed for BIBE because no clear trend in $\Delta c_{p}(T)$ could be detected in the present HCS frequency window. The following methods are used.

(i) Find a $T_{\text {on }}$ temperature and a $\omega_{\text {on }}$ frequency from the intersection of dielectric $\alpha$ and $\beta$ traces in the Arrhenius plot.

(ii) Find a peculiarity in the temperature dependence of $d / d T\left[\left(d \log \omega_{\max } / d T\right)^{-1 / 2}\right]$ for the dielectric trace (Stickel method $\left.{ }^{18}\right)$.

(iii) Similarly, from $(-d \log \eta / d(1 / T))^{-1 / 2}$ for the viscosity.

(iv) Find $\log \omega_{\mathrm{on}}$ from a peculiarity of the $\Delta \varepsilon$ vs $\log \omega$ plot (Schönhals et al. method ${ }^{30}$ ), and find then the corresponding $T_{\text {on }}$ temperature from the Arrhenius plot.

(v) Find the $\nu_{g}\left(T_{\text {on }}\right)$ function from linear extrapolations of $N_{\alpha}^{v_{g}} \sim\left(T_{\text {on }}-T\right)$ to $N_{\alpha} \rightarrow 0$ from HCS.

In the Appendix two further methods [(vi) and (vii)] to determine onset temperatures $T_{\text {on }}$ are described.

The aim of this paper is thus to find a consistent set of cooperativity onset parameters: temperature $T_{\text {on }}$, frequency $\omega_{\text {on }}$, and cooperativity exponent $\nu_{g}$, from viscosity, dielectric spectroscopy, and heat capacity spectroscopy HCS for benzoin isobutylether.

\section{EXPERIMENT}

\section{A. Sample}

The benzoin isobutylether sample (BIBE) was purchased from Aldrich. It was purified by distillation under reduced pressure over a short column. $\left[b p(2 \mathrm{mbar})=154^{\circ} \mathrm{C}\right]$. $100 \mathrm{ml}$ of the raw product yielded about $85 \mathrm{ml}$ of the yellow, viscose liquid. BIBE is a photo initiator and has a weak tendency to crystallization (as observed, e.g., after many weeks at a storage temperature of about $-18^{\circ} \mathrm{C}$ ). The crystallization rate of the unpurified sample is slightly higher. The dipole moment of one BIBE molecule is about 2D, as estimated from charge distribution and hybridization.

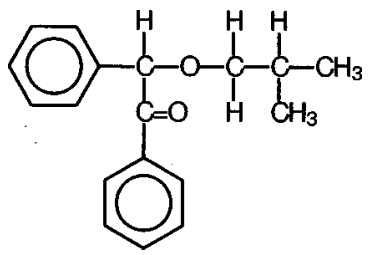

benzoin isobutylether (BIBE) 


\section{B. Measurements}

\section{Dielectric spectroscopy}

The dielectric measurements were carried out on two different setups to cover a broad frequency range $(\omega=2 \pi$ $\times 10^{-3} \mathrm{rad} / \mathrm{s}$ to $\omega=2 \pi \times 2 \times 10^{9} \mathrm{rad} / \mathrm{s}$ ). A broadband dielectric spectrometer Novocontrol BDS 4000 (based on a Schlumberger 1260 frequency response analyzer) was used to measure the dielectric function, $\varepsilon^{*}(\omega)=\varepsilon^{\prime}(\omega)$ $-i \varepsilon^{\prime \prime}(\omega)$, in the frequency range from $\omega=2 \pi \times 10^{-3} \mathrm{rad} / \mathrm{s}$ to $\omega=2 \pi \times 10^{7} \mathrm{rad} / \mathrm{s}$. The high frequency range $(\omega=2 \pi$ $\times 10^{6} \mathrm{rad} / \mathrm{s}$ to $\omega=2 \pi \times 2 \times 10^{9} \mathrm{rad} / \mathrm{s}$ ) was covered by a Novocontrol BDS 6000 (based on a Hewlett Packard 4291 impedance analyzer). Isothermal frequency scans were performed in the temperature range $-130{ }^{\circ} \mathrm{C}$ to $+55^{\circ} \mathrm{C}$.

To perform the Stickel method ${ }^{18}$ we measured the dielectric data in the temperature range -50 to $+55^{\circ} \mathrm{C}$ with small temperature steps. First, downwards, isothermal frequency scans with temperature steps of $1 \mathrm{~K}$ were taken. Subsequently, beginning at the lowest temperature with a shift of $0.5 \mathrm{~K}$, isothermal frequency scans in $1 \mathrm{~K}$ steps upwards were taken. This allows to check the dielectric data for possible influence of thermal history. No significant influence on the peak positions could be detected. The dielectric intensity also remained stable within $0.3 \%$.

Using the nonlinear least-squares Levenberg-Marquard method, ${ }^{33}$ in the range -50 to $+55^{\circ} \mathrm{C}$ the dielectric results are fitted by single Havriliak-Negami (HN) function ${ }^{34}$

$\varepsilon^{*}(\omega)=\varepsilon^{\prime}(\omega)-i \varepsilon^{\prime \prime}(\omega)=\Delta \varepsilon \cdot\left(1+\left(i \omega / \omega_{c}\right)^{b}\right)^{-g}+\varepsilon_{\infty}$

and a conductivity term $i \sigma_{\mathrm{DC}} / \varepsilon_{0} \omega^{-A},{ }^{35}$ where $\sigma_{\mathrm{DC}}$ is the temperature dependent $\mathrm{DC}$ conductivity and $A=1$ (in our case) means ionic or ohmic conductivity. $\varepsilon_{\infty}$ is the high frequency limit of $\varepsilon^{\prime}$ outside the dispersion zone. Because of the small magnitude of the $\beta$-process, it is not possible to separate $\alpha$ and $\beta$ process $\mathrm{HN}$-functions from a common $\varepsilon^{\prime \prime}(\log \omega)$ peak in the crossover region.

The dielectric results below $T_{g}$ are fitted by Cole-Cole functions for $\beta$ and $\gamma$ relaxation, respectively [parameter $g$ in Eq. (7) was set to one] and a power law (to consider the high frequency wing of the $\alpha$ process). Usually, local processes are well represented by Cole-Cole functions. ${ }^{36}$

The maximum loss frequency $\omega_{\max }$ was analytically calculated from the fit parameters by

$$
\omega_{\max }=\omega_{c}\left[\frac{\sin \frac{\pi}{2} b}{\tan \frac{\pi b}{2(g+1)}}-\cos \frac{\pi}{2} b\right]^{-1 / b} .
$$

For $\omega \ll \omega_{\max }$ and $\omega \gg \omega_{\max }$ the HN function for $\varepsilon^{\prime \prime}$ reduces to power laws with exponents $b$ and $-b g,{ }^{37}$ respectively.

\section{Viscosity measurements}

Three rheometers, RDA II, RFS II, and DSR (Rheometrics), were used to determine the temperature dependence of viscosity in the temperature range $-46^{\circ} \mathrm{C}$ to $+60^{\circ}$. In the RFS and DSR analyzer we used a Couette (RFS: cup diameter $=34 \mathrm{~mm}$, bob diameter $=32 \mathrm{~mm}$, length $=33.5 \mathrm{~mm}$,
DSR: cup diameter $=32 \mathrm{~mm}$, bob diameter $=29.5 \mathrm{~mm}$, length $=44 \mathrm{~mm}$ ), and in RDA parallel plate geometry (plate diameter $=25 \mathrm{~mm}$, gap $\approx 1 \mathrm{~mm}$ ).

\section{Heat capacity spectroscopy (HCS)}

The frequency dependence of the product of mass density, thermal conductivity, and heat capacity, $\left(\rho \kappa c_{p}(\omega)\right)^{*}$, is measured by the $3 \omega$ method $^{38}$ using a heat capacity spectrometer according to Birge and Nagel. The product was decoupled assuming that the thermal conductivity $\kappa$ and density $\rho$ have no dispersion, ${ }^{39}$ i.e., $\left(\rho \kappa c_{p}(\omega)\right)^{*}=\rho \kappa c_{p}^{\prime}(\omega)$ $-i \rho \kappa c_{p}^{\prime \prime}(\omega)$.

The central part of the spectrometer is a thin-film $(70 \cdots 100 \mathrm{~nm})$ nickel heater on the surface of a thick $(4 \mathrm{~mm})$ substrate of poly(ether ether ketone), PEEK. The lateral dimensions of the nickel stripe are about $1 \times 5 \mathrm{~mm}^{2}$. The heater has a high temperature coefficient of electrical resistance $\left(\approx 1 \cdots 2 \times 10^{-3} \mathrm{~K}^{-1}\right)$ and is also used as the thermometer. This assembly was coated with the sample after a reference measurement at different frequencies for the determination of the heater and substrate parameters. The sample thickness was about $4 \mathrm{~mm}$. Further details of the spectrometer are published in Ref. 32. We started at the highest temperature $\left(T=30^{\circ} \mathrm{C}\right)$, the equilibration time before each frequency sweep was $900 \mathrm{sec}$.

\section{Differential scanning calorimetry (DSC) and temperature modulated DSC (TMDSC)}

A DSC 7 apparatus (Perkin Elmer), was used with cooling and heating rates of $|d T / d t|=10 \mathrm{~K} / \mathrm{min}$ in the temperature interval from $0{ }^{\circ} \mathrm{C}$ down to $-100{ }^{\circ} \mathrm{C}$, and annealing times of $5 \mathrm{~min}$ at $T=-100{ }^{\circ} \mathrm{C}$. The TMDSC curve was recorded with saw-tooth waves and a frequency $\nu=\omega / 2 \pi$ $=16.7 \mathrm{mHz}$. The amplitude of the first harmonic was $0.2 \mathrm{~K}$, and the underlying cooling rate was $0.5 \mathrm{~K} / \mathrm{min}$. The temperature interval for a scan was from $-10^{\circ} \mathrm{C}$ down to $-90^{\circ} \mathrm{C}$. The sample weight was about $5.5 \mathrm{mg}$.

The glass temperature $T_{g}$ was calculated by an equalarea construction using the two tangents below and above $T_{g} \cdot{ }^{40}$

\section{RESULTS}

The TMDSC curves for the purified sample with a period $\tau_{p}=60 \mathrm{~s}(\nu=16.7 \mathrm{mHz})$ show the usual step in the real part, $c_{p}^{\prime}(T)$, and the usual maximum in the imaginary part, $c_{p}^{\prime \prime}(T)$ [Fig. 2(A)]. The Gaussian fit Eq. (2) results in the maximum temperature $T_{\nu}=-49^{\circ} \mathrm{C}$ and the dispersion $\delta T$ $=2.7 \mathrm{~K}$. The glass temperature from the DSC heating thermogram at $10 \mathrm{~K} / \mathrm{min}$ [Fig. 2(B)] is $T_{g}=-53{ }^{\circ} \mathrm{C}$ for both the purified and unpurified sample. The DSC melting point of a partially crystallized sample (stored more than 30 days at $T$ $=-18^{\circ} \mathrm{C}$ ) is about $T_{m}=+40^{\circ} \mathrm{C}$ [inset of Fig. 2(B)].

The imaginary part of dielectric function, $\varepsilon^{\prime \prime}(\log \omega)$, for temperatures from $-110^{\circ} \mathrm{C}$ to $+50{ }^{\circ} \mathrm{C}$, displays three relax 


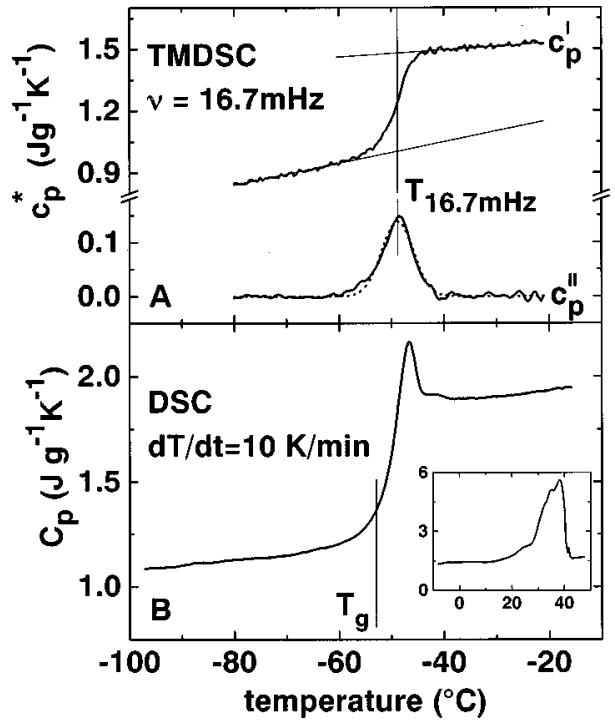

FIG. 2. Specific heat capacity of benzoin isobutylether (BIBE, purified sample) in the glass transition region from TMDSC (A) and DSC (B). The dotted line in the imaginary part $c_{p}^{\prime \prime}$ is a Gaussian fit by Eq. (2). The inset in (B) shows the melting curve of a partially crystallized sample. The melting point is at about $T_{m}=40^{\circ} \mathrm{C}$.

ation processes: $\alpha, \beta$, and $\gamma$ [Figs. 3(A) and 4]. The $\alpha$ intensity is more than 100 times larger than the $\beta$ and $\gamma$ intensities. An $\alpha \beta$ separation analysis using an additive ansatz $\varepsilon_{\alpha}^{*}+\varepsilon_{\beta}^{* 24,25}$ would, therefore, fail in the crossover region.

The complex dielectric relaxation behavior was analyzed at lower temperatures where the $\alpha$ relaxation is separated from the $\beta$ and $\gamma$ relaxations. Figure 4 shows examples for the dielectric traces, the fit functions, and the deviations between measurement and fit for three representative temperatures. At $T=-110^{\circ} \mathrm{C}$ we have a separate peak for the $\beta$ process, at $T=-90^{\circ} \mathrm{C}$ the high frequency $\alpha$ wing superimposes the $\beta$ process. For temperatures $T>-65^{\circ} \mathrm{C}$ a meaningful analysis of the $\beta$ process is no longer possible.
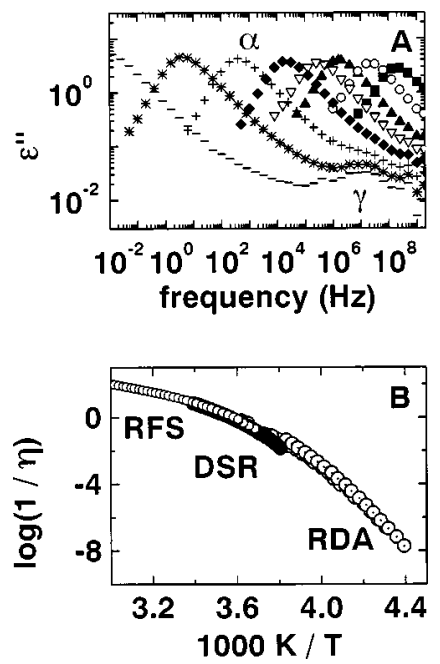

FIG. 3. (A) Imaginary part of dielectric loss $\varepsilon^{\prime \prime}$ as a function of frequency at 8 selected, high temperatures $\left(\boldsymbol{\square}, 50{ }^{\circ} \mathrm{C} ; \bigcirc, 20^{\circ} \mathrm{C} ; \boldsymbol{\Delta}, 0{ }^{\circ} \mathrm{C} ; \nabla,-10{ }^{\circ} \mathrm{C} ; \bullet\right.$, $-20{ }^{\circ} \mathrm{C} ;+,-30{ }^{\circ} \mathrm{C} ;{ }^{*},-40{ }^{\circ} \mathrm{C} ;---, 50^{\circ} \mathrm{C}$ ) (only every third point is shown) for the purified sample. (B) Logarithm of reciprocal viscosity vs reciprocal temperature for the purified sample from different devices: O, RFS; DSR; $\odot$, RDA.

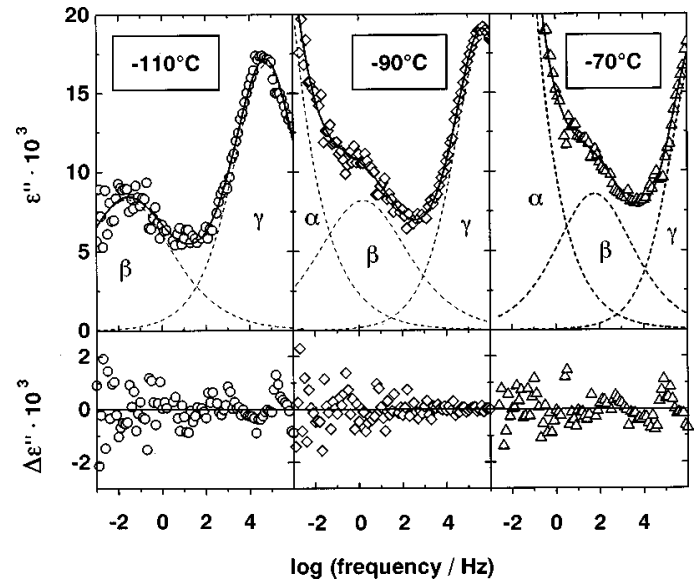

FIG. 4. Imaginary part of dielectric loss $\varepsilon^{\prime \prime}$ as a function of frequency at three selected, low temperatures. The solid line in the isotherms results from a two Cole-Cole function fit and the dotted lines correspond to each component of the fit. The lower part of the figures shows the deviation plot between measured values and fit curve.

The peak locations for the different processes were transferred to an Arrhenius diagram (Fig. 6, see below). The activation energy of the $\beta$ process is about $E_{A}^{\beta}=55 \mathrm{~kJ} / \mathrm{mol}$ and the extrapolated frequency asymptote $\log \left(\Omega^{\beta} / \mathrm{rad} \mathrm{s}^{-1}\right)$ $=16.3 \pm 1$. The activation energy of the $\gamma$ process is about $E_{A}^{\gamma}=28 \mathrm{~kJ} / \mathrm{mol}$ and the extrapolated frequency asymptote $\log \left(\Omega^{\gamma} / \mathrm{rad} \mathrm{s}^{-1}\right)=14.3 \pm 1$.

The shear viscosity depends on temperature as expected for a fragile liquid [Fig. 3(B)]. The extrapolated steepness index at $T=T_{g}$ (DSC) is $m=-d \log _{10} \eta / d \ln T=100$. The parameters of the two separate $\mathrm{VFT}^{41}$ equations

$$
\log \left(\eta / \eta_{0}\right)=B^{\prime} /\left(T-T_{\infty}^{\prime}\right)
$$

above and below $T=-8{ }^{\circ} \mathrm{C}$ are listed in Table I. The extrapolated high temperature viscosity $\log \left(\eta_{0} / \mathrm{Pa} s\right)$ is -3.9 \pm 1 for the $a$ and $-7.4 \pm 1$ for the $\alpha$ process.

Deviations between different measurements of viscosity in the temperature region below about $0{ }^{\circ} \mathrm{C}$ of Fig. 3(B) were observed. Surprisingly, we found a small real part (order 1 per cent) of the shear modulus there. The reason could not be clarified. Note that this region corresponds to the crossover. The temperature derivatives used below are less affected [see Fig. 7(B), below].

Isothermal HCS sweeps for seven frequencies between $\nu=\omega / 2 \pi=2 \mathrm{~Hz}$ and $\nu=2000 \mathrm{~Hz}$ yield the complex quantity $\rho \kappa c_{p}^{*}$ (Fig. 5). The real part shows the expected $c_{p}^{\prime}(T)$ step [Fig. 5(A)] and the imaginary part shows the expected $c_{p}^{\prime \prime}(T)$ peak [Fig. 5(B)]. Both, peak and step, shift to higher fre-

TABLE I. VFT parameters for $a$ and $\alpha$ traces (Fig. 6) from dielectric and viscosity measurements.

\begin{tabular}{cllll}
\hline \hline Process & \multicolumn{1}{c}{$a$} & \multicolumn{1}{c}{$\alpha$} \\
\hline Parameter & $B(\mathrm{~K})$ & $T_{\infty}\left({ }^{\circ} \mathrm{C}\right)$ & $B(\mathrm{~K})$ & $T_{\infty}\left({ }^{\circ} \mathrm{C}\right)$ \\
Dielectric & $305 \pm 30$ & $-61 \pm 10$ & $683 \pm 20$ & $-93 \pm 10$ \\
Viscosity & $208^{\mathrm{a}}$ & $-48 \pm 10$ & $724 \pm 20$ & $-93 \pm 10$ \\
\hline \hline
\end{tabular}

${ }^{\mathrm{a}}$ Influenced by systematic reasons. See Fig. 3(B) and text. 


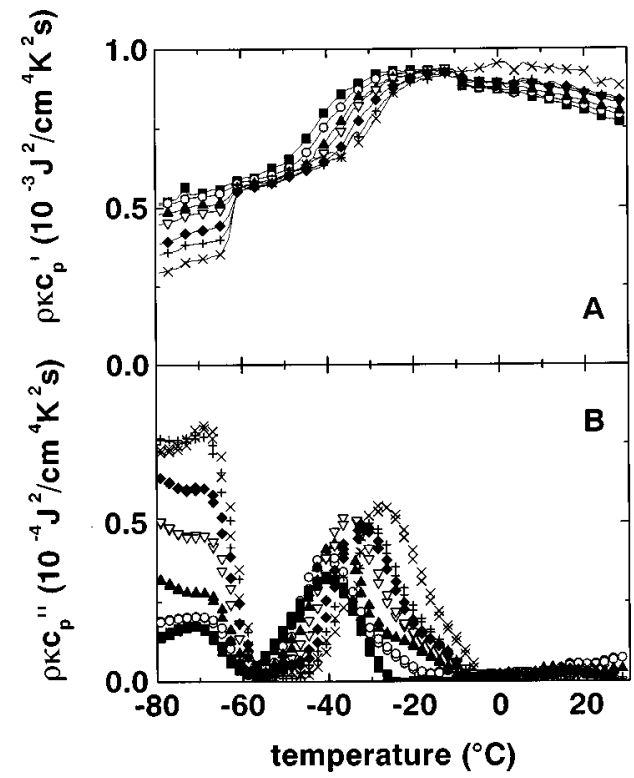

FIG. 5. Real (A) and imaginary part (B) of $\rho \kappa c_{p}^{*}$ versus temperature from HCS at seven frequencies $(\boldsymbol{\square}, 2 \mathrm{~Hz} ; \bigcirc, 6 \mathrm{~Hz} ; \boldsymbol{\Lambda}, 20 \mathrm{~Hz} ; \nabla, 60 \mathrm{~Hz} ;, 204$ $\mathrm{Hz} ;+, 600 \mathrm{~Hz} ; \times, 2000 \mathrm{~Hz}$ ) for the purified sample. The temperature step was $2 \mathrm{~K}$, and $\rho \kappa c_{p}^{*}$ was measured two times at each temperature. The real part was used to estimate the heat capacity step height. For reasons of clarity only each fourth point is shown. The imaginary part was used to estimate the dispersion $\delta T$ and peak maximum temperature $T_{\nu}$ [via Gaussian fit Eq. (2)]. Each isochronous measurement was averaged (8 points) and FFT filter smoothed. The disturbance at about $T=-60^{\circ} \mathrm{C}$ results from contact problems between the sample and nickel heater.

quencies with increasing temperature. The step at about $T$ $=-60{ }^{\circ} \mathrm{C}$ is an artifact generated by partial separation of the sample from the nickel heater.

The parameters of the HCS sweeps (two separate runs with the purified and unpurified sample, respectively) are

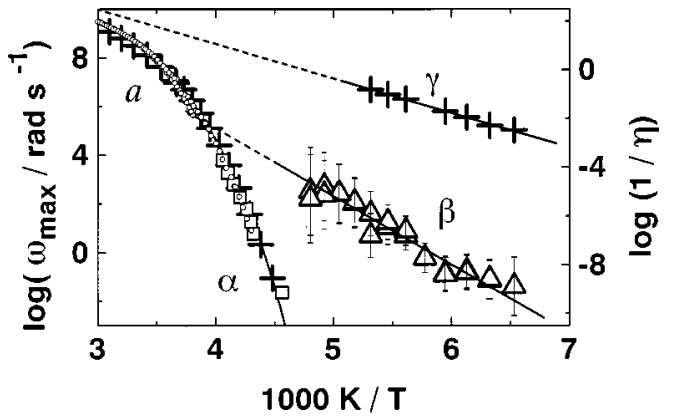

FIG. 6. Arrhenius diagram for the purified BIBE sample. The frequency position of the peak maxima of $c_{p}^{\prime \prime}(\square)$, dielectric $\alpha(+), \beta(\triangle)$, and $\gamma(+)$ process $\varepsilon^{\prime \prime}$ correspond to the left axis. The TMDSC peak maximum is included. The viscosities $(\bigcirc)$ correspond to the right axis.

listed in Table II. The $c_{p}^{\prime \prime}(T)$ peaks were fitted with the Gaussian of Eq. (2) defining the maximum temperatures $T_{\nu}$ and the temperature dispersions $\delta T$. The $c_{p}$ steps heights, $\Delta c_{p}$, and the $\bar{c}_{p}$ average of glassy and liquid zones at the $T_{\nu}$ maximum temperature are obtained from a tangent construction of $c_{p}^{\prime}(T) .{ }^{10,25}$ Each isochronous $c_{p}^{\prime}(T)$ curve was calibrated at $T=-10^{\circ} \mathrm{C}$ by the DSC curve.

\section{DISCUSSION}

The discussion follows the method listing at the end of the Introduction.

(i) The Arrhenius diagram $\left(\log \omega_{\max }\right.$ or $\log 1 / \eta$ vs $\left.1 / T\right)$ includes all the different physical quantities discussed in this paper (Fig. 6). The $\beta$ and $\gamma$ traces are only indicated by dielectrics. The linear $\beta$ extrapolation intersects the dielectric $\alpha$ trace at $\log _{10}\left(\omega_{\text {on }} / \mathrm{rad} \mathrm{s}^{-1}\right)=5.5 \pm 1.0$ and $T_{\text {on }}$ $=-17^{\circ} \mathrm{C} \pm 10 \mathrm{~K}$. The $\alpha$ traces of calorimetry and dielec-

TABLE II. Calorimetric characteristics of dynamic glass transition in BIBE at different frequencies, $\nu$, from HCS for two different measurements. The $16.7 \mathrm{mHz}$ isochronous data from TMDSC and the $\dot{T}=10 \mathrm{~K} / \mathrm{min}$ DSC heating run data are included. $T_{\nu}=$ temperature of the $c_{p}^{\prime \prime}(T)$ peak maximum for each isochron, $\Delta c_{p}$ the heat capacity step height, $\bar{c}_{p}=\left(c_{p}^{l}+c_{p}^{g}\right) / 2$ at $T_{\nu}$, with $c_{p}^{l}$ and $c_{p}^{g}$ the heat capacity at $T_{\nu}$ extrapolated from liquid and glass zone, $\delta T=$ temperature dispersion from a Gauss fit of the isochronous $c_{p}^{\prime \prime}(T)$ peaks, $c_{p}^{\prime \prime}(T) \sim \exp (-(T$ $\left.\left.-T_{\nu}\right)^{2} / 2(\delta T)^{2}\right), N_{\alpha}=$ cooperativity calculated from Eq. (1).

\begin{tabular}{rcccrr}
\hline \hline$\nu / \mathrm{Hz}$ & $T_{\nu} /{ }^{\circ} \mathrm{C}$ & $\Delta c_{p} / \mathrm{J} \mathrm{g}^{-1} \mathrm{~K}^{-1}$ & $\overline{c_{p}} / \mathrm{J} \mathrm{g}^{-1} \mathrm{~K}^{-1}$ & $\delta T / \mathrm{K}$ & $N_{\alpha}$ \\
\hline $2000^{\mathrm{a}}$ & -26.7 & 0.37 & 1.23 & 15.2 & 6 \\
$600^{\mathrm{a}}$ & -29.4 & 0.41 & 1.23 & 15.0 & 7 \\
$204^{\mathrm{a}}$ & -32.2 & 0.44 & 1.23 & 13.9 & 9 \\
$60^{\mathrm{a}}$ & -35.3 & 0.47 & 1.23 & 12.2 & 12 \\
$20^{\mathrm{a}}$ & -38.4 & 0.48 & 1.23 & 12.4 & 12 \\
$6^{\mathrm{a}}$ & -40.6 & 0.48 & 1.23 & 11.3 & 14 \\
$2^{\mathrm{a}}$ & -41.6 & 0.47 & 1.23 & 11.0 & 14 \\
$2000^{\mathrm{b}}$ & -33.0 & 0.37 & 1.36 & 15.3 & 8 \\
$600^{\mathrm{b}}$ & -36.0 & 0.43 & 1.35 & 9.8 & 21 \\
$204^{\mathrm{b}}$ & -39.3 & 0.49 & 1.34 & 9.8 & 23 \\
$60^{\mathrm{b}}$ & -41.9 & 0.51 & 1.36 & 9.6 & 25 \\
$20^{\mathrm{b}}$ & -44.3 & 0.54 & 1.35 & 7.5 & 41 \\
$6^{\mathrm{b}}$ & -47.2 & 0.57 & 1.36 & 7.8 & 39 \\
$2^{\mathrm{b}}$ & -50.1 & 0.54 & 1.42 & 7.9 & 34 \\
$0.0167^{\mathrm{b}}$ & -49 & 0.48 & 1.25 & 2.7 & 65 \\
$\dot{T}=10 \mathrm{~K} /$ min $^{\mathrm{b}}$ & -53 & 0.72 & $\pm 10 \%$ & 2.2 & 100 \\
Uncertainty $^{\mathrm{b}}$ & \pm 2 & $\pm 10 \%$ & $\pm 20 \%$ & $\pm 40 \%$ \\
\hline \hline
\end{tabular}

${ }^{\mathrm{a}}$ For unpurified sample.

${ }^{\mathrm{b}}$ For purified sample. 

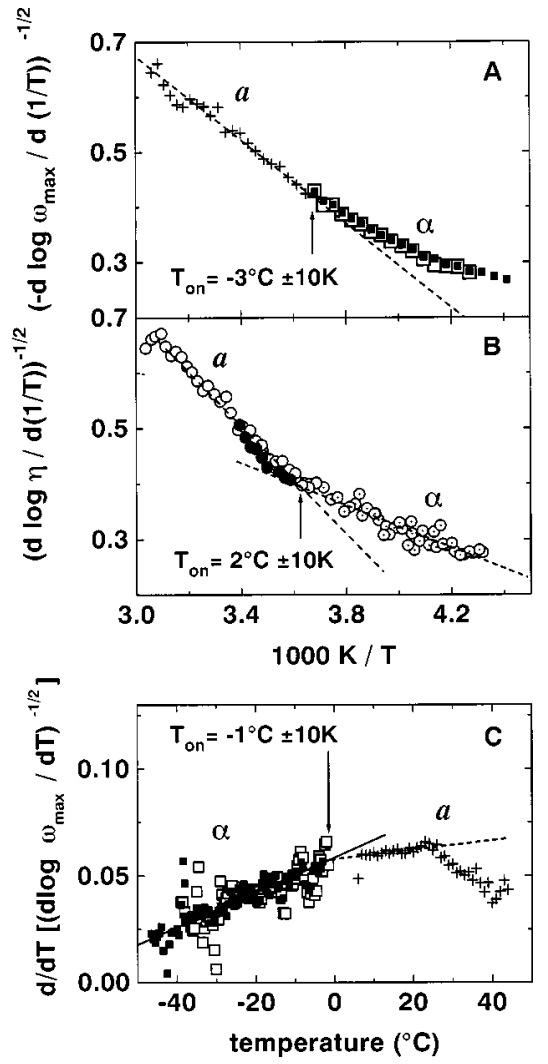

FIG. 7. Temperature dependence of different temperature derivatives for BIBE: $(-d \log x / d(1 / T))^{-1 / 2}$ versus $1 / T$, where $x$ represents $\omega_{\max }$ [dielectric measurements, for the purified $(\square,+)$ and unpurified sample $(\boldsymbol{\square})(\mathrm{A})$, and $1 / \eta$ for the purified sample (B)]. The viscosities where measured with RDA $(\odot)$, RFS $(\bigcirc)$, and DSR $(\odot)$. (C) Second derivative of dielectric data of part (A).

trics coincide within the experimental uncertainty. The temperature dependence of viscosity nearly parallels those of the dielectric $\alpha$ trace.

(ii) and (iii) The first derivative ${ }^{18}$ of the dielectric $\alpha$ trace seems to indicate a peculiarity at $T_{\text {on }}=-3{ }^{\circ} \mathrm{C} \pm 10 \mathrm{~K}$ [Fig. 7(A)], corresponding to $\log \left(\omega_{\mathrm{on}} / \mathrm{rad} \mathrm{s}^{-1}\right)=6.7 \pm 1.0$ (from Fig. 6). This peculiarity separates the high-temperature $a$ process from the low-temperature $\alpha$ process. The derivative is calculated by a two-point secant between the right and left neighbor points. The first derivative of viscosity (Fig. 7B) indicates the onset peculiarity at $T_{\text {on }}=2{ }^{\circ} \mathrm{C} \pm 10 \mathrm{~K}$, corresponding to $\log \left(\omega_{\text {on }} / \mathrm{rad} \mathrm{s}^{-1}\right)=7.1 \pm 1.0$. As mentioned above, the VFT parameters for $\alpha$ and $a$ processes are listed in Table I. The second derivative of the dielectric trace, according to Stickel, ${ }^{18}$ shows a bend at $T_{\text {on }}=-1{ }^{\circ} \mathrm{C} \pm 10 \mathrm{~K}$ [Fig. 7(C)], corresponding to $\log \left(\omega_{\text {on }} / \mathrm{rad} \mathrm{s}^{-1}\right)=6.7 \pm 1.0$. The second derivative would be constant for a WLF equation, $\quad d / d T\left[\left(d \log \omega_{\max } / d T\right)^{-1 / 2}\right]=B^{-1 / 2}=0.037$. The slopes in Fig. 7(C) are different from zero indicating small but definite deviations from WLF equations for $a$ and $\alpha$. The physical nature of the second bend at about $T=25^{\circ} \mathrm{C}$ is not known. Note that the $\gamma$ process extrapolation does not intersect the $a$ process at this temperature (Fig. 6). The values for the frequency asymptote $\log \left(\Omega / \mathrm{rad} \mathrm{s}^{-1}\right)$ are $11.8 \pm 1$ for the $a$ and $14.4 \pm 1$ for the $\alpha$ process.

(iv) The dielectric Schönhals plot ( $\log \omega_{\max }$ vs $\Delta \varepsilon_{\alpha}$, the dielectric intensity $\Delta \varepsilon_{\alpha}$ ) has a weak bend at $\log _{10}\left(\omega_{\mathrm{on}} / \mathrm{rad} \mathrm{s}^{-1}\right)=5.9 \pm 1.0$ corresponding, via the projection on an Arrhenius plot, to $T_{\text {on }}=-13{ }^{\circ} \mathrm{C} \pm 10 \mathrm{~K}$ [Fig. 8(A) and 8(B)]. Additionally, two WLF curves for the regions above and below the crossover calculated from the parameters of Table I are included in Fig. 8(B).

(v) We calculate now the function $\nu_{g}\left(T_{\text {on }}\right)$ from a linear extrapolation of $N_{\alpha}^{\nu_{g}}$ [Eq. (6)] to zero (this results in $T$ $=T_{\text {on }}$ for given $\nu_{g}$ ). Some examples are given in Fig. 9(A) $\left(\nu_{g}=0.3 / 0.5 / 1.0\right)$. The full function [Fig. 9(B)] is used to determine the cooperativity exponent $\nu_{g}$ from $T_{\text {on }}$ for each method, (i)...(iv). The results are listed in Table III. The average value is $\nu_{g}=0.49 \pm 0.15 \approx 1 / 2$. Two further variants [(vi) and (vii); see Appendix] are also displayed.

Finally, $\Delta c_{p}(T)$ and $N_{\alpha}^{1 / 2}(T)$ are directly displayed as a function of temperature (Fig. 10). The $\Delta c_{p}$ values from the tangent construction of $c_{p}^{\prime}(T)$ where supplemented by isochronous $\Delta c_{p}$ values calculated from $c_{p}^{\prime \prime}(T)$ using the Kramers-Kronig relation ${ }^{42}$

$$
\Delta c_{p}(T)=(2 / \pi) \cdot \ln 10 \cdot d \log \omega / d T(T) \cdot \int_{\text {Peak }} c_{p}^{\prime \prime}(T) \cdot d T .
$$

No clear $\Delta c_{p}(T)$ trend can be predicted [Fig. 10(A)]. The parameter with the strongest temperature dependence is $\delta T$. The square root of cooperativity, correspondingly, varies between about 6 and 2, and shows a clear trend to zero in the crossover. The cooperativity $N_{\alpha}$ itself varies between 39 and 6 (Table II), i.e., more than a factor of six. The cooperativity exponent $\nu_{g}=1 / 2$ as used in Fig. 10(B) leads, via a linear

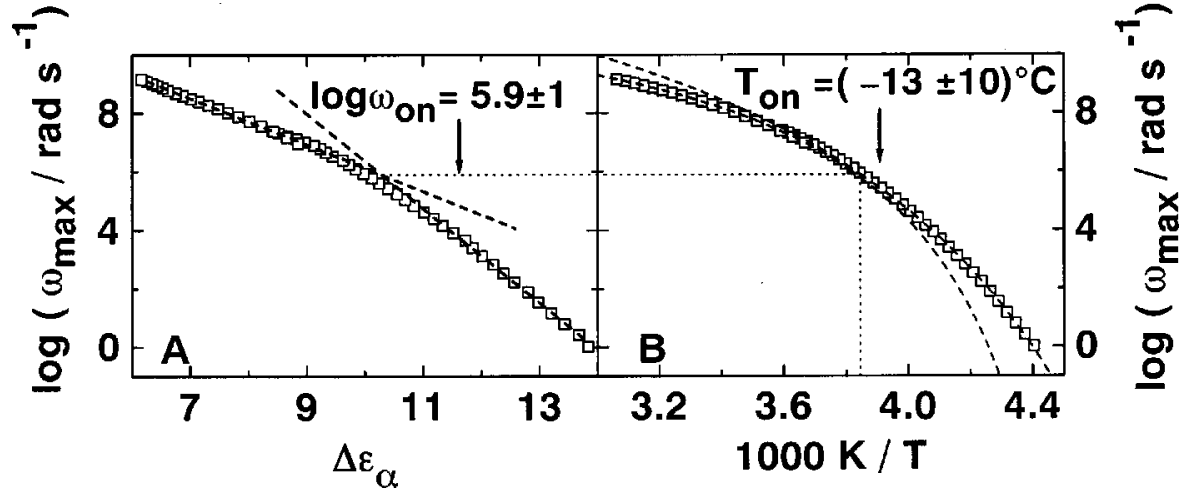

FIG. 8. Dielectric peak maximum frequency $\log \omega_{\max }$ vs dielectric relaxation strength $\Delta \varepsilon_{\alpha}$ for the purified sample (A). The tangents indicate two temperature regimes of the dynamic glass transition. The extrapolated crossover of the tangents is at $\log \left(\omega_{\max } / \mathrm{rad} \mathrm{s}^{-1}\right)=5.9 \pm 1.0, \quad$ corresponding to a temperature of about $-13{ }^{\circ} \mathrm{C} \pm 10 \mathrm{~K}$ in an Arrhenius diagram (B). The WLF traces, touchingnot crossing - each other in the crossover, from the parameters of Table I are included in the Arrhenius diagram. 

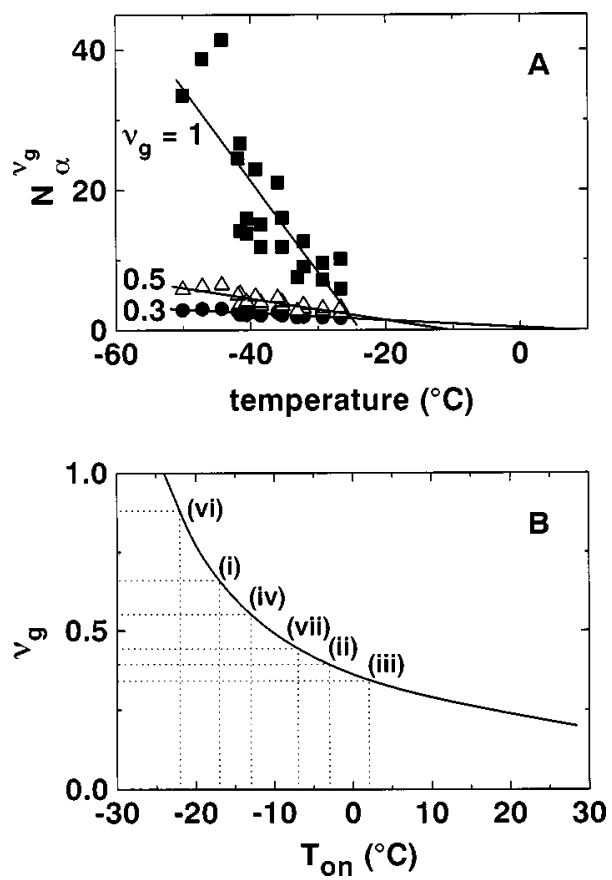

FIG. 9. (A) Temperature dependence of $N_{\alpha}^{\nu_{g}}$ for different examples: $\nu_{g}=1$ (匹), $\nu_{g}=0.5(\triangle), \nu_{g}=0.3(\bullet)$. The linear fits $N_{\alpha}^{v_{g}}(T) \rightarrow 0$ mark the onset temperatures $T_{\text {on }}$ for different $\nu_{g}$ defining the $\nu_{g}\left(T_{\text {on }}\right)$ function [method (v)]. (B) Exponent $\nu_{g}$ versus the extrapolated onset temperatures $T_{\text {on }}$. The dotted lines indicate the values from the other methods listed in the Introduction [(i): $\alpha \beta$ crossover in the Arrhenius diagram, Fig. 6 ; (ii): dielectric peculiarity, Fig. 7(A); (iii): peculiarity in the viscosity, Fig. 7(B); (iv): peculiarity in the $\log \omega_{\max }$ vs $\Delta \varepsilon$ plot, Fig. 8; (vi): $d x / d T=0$, Fig. 13; and (vii): $d x^{\prime} / d T$ $=0$, Fig. 14, both in the Appendix). The different $T_{\text {on }}$ values correspond to different exponent $\nu_{g}$ values.

extrapolation, to the onset temperature $T_{\text {on }}=-11^{\circ} \mathrm{C}$ $\pm 10 \mathrm{~K}$, consistent with the average value obtained with the four methods above.

\section{CONCLUSIONS}

From a combination of the four evaluation methods listed at the end of the Introduction we find the following consistent crossover $=$ onset parameters for benzoin isobutylether:

TABLE III. Crossover temperatures $T_{\text {on }}$, corresponding frequencies $\log \omega_{\text {on }}$, and cooperativity exponent $\nu_{g}$ for different methods of data analysis. The bold roman numerals indicate the method (see the Introduction and Fig. 9).

\begin{tabular}{llccc}
\hline \hline & \multicolumn{1}{c}{ Method } & $T_{\text {on }}\left({ }^{\circ} \mathrm{C}\right)$ & $\log \left(\omega_{\mathrm{on}} / \mathrm{rad} \mathrm{s}^{-1}\right)$ & $\nu_{g}$ \\
\hline (i) & $\alpha \beta$ crossover & -17 & 5.5 & 0.66 \\
(ii) & Stickel $\varepsilon^{*}$ & -3 & 6.7 & 0.41 \\
(iii) & Stickel $\eta$ & 2 & 7.1 & 0.36 \\
(iv) & Schönhals $\Delta \varepsilon$ & -13 & 5.9 & 0.49 \\
(v) & $N_{\alpha}^{1 / 2} \rightarrow 0$ & -11 & 6.2 & $(1 / 2)$ \\
(vi) & $d x / d T=0^{\mathrm{a}}$ & -22 & 4.8 & 0.88 \\
(vii) & $d x^{\prime} / d T=0^{\mathrm{b}}$ & -7 & 6.5 & 0.48 \\
& Uncertainty $^{\mathrm{c}}$ & $\pm 10 \mathrm{~K}$ & \pm 1 & $\pm 25 \%$ \\
& Average & $-8 \pm 8 \mathrm{~K}$ & $6.3 \pm 0.7$ & $0.49 \pm 0.15$ \\
\hline \hline
\end{tabular}

${ }^{\mathrm{a}} x=\delta T \times\left(T-T_{\mathrm{on}}\right)^{1 / 2}$

${ }^{\mathrm{b}} x^{\prime}=\delta T \times\left(T-T_{\text {on }}\right)$.

${ }^{\mathrm{c}}$ From methods (i) $\cdots($ iv).

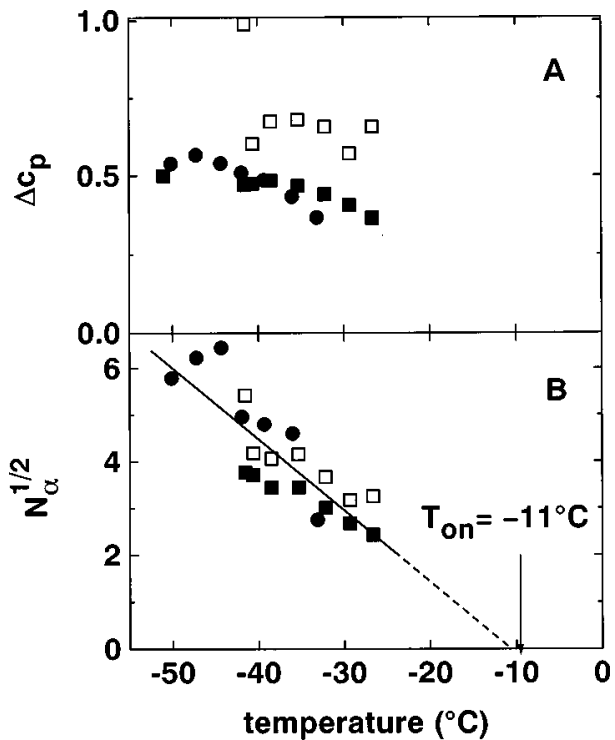

FIG. 10. Heat capacity step height $\Delta c_{p}$ (A) and square root of cooperativity $N_{\alpha}^{1 / 2}(\mathrm{~B})$ as a function of temperature for the purified ( $\square$ ) and unpurified (O) sample. The $\square$ symbols result from a Kramers-Kronig evaluation of the imaginary part for the purified sample. The solid line is a linear fit, and the extrapolated onset temperature is $T_{\text {on }}=-11^{\circ} \mathrm{C} \pm 10 \mathrm{~K}$.

$$
\begin{aligned}
& T_{\text {on }}=-8{ }^{\circ} \mathrm{C} \pm 8 \mathrm{~K}, \\
& \log _{10}\left(\omega_{\text {on }} / \mathrm{rad} \mathrm{s}^{-1}\right)=6.3 \pm 0.7, \\
& \nu_{g}=0.49 \pm 0.15
\end{aligned}
$$

The \pm values indicated include possible systematic uncertainties. It seems that the confidence is high enough to conclude that BIBE has a cooperativity onset in the crossover region which is consistent with Eq. (5), $N_{\alpha}^{1 / 2} \sim\left(T_{\text {on }}\right.$ $-T$ ), for $T<T_{\text {on }}-10 \mathrm{~K}$. An enlargement of the HCS frequency window could improve the confidence in the future.

Since BIBE belongs to the dielectric II scenario and since Eq. (5) is typical for the dielectric I scenario, our results suggest a similar cooperativity onset in both crossover scenarios, I and II. We think that such a cooperativity onset is an important and general characteristics of the crossover region of dynamic glass transition. For the few substances accessible by heat capacity spectroscopy so far, the hightemperature $a$ relaxation above the onset has only a small $N_{\alpha} \approx 1$ cooperativity. The low-temperature $\alpha$ relaxation below the crossover has an increasing cooperativity reaching $N_{\alpha} \approx 100$ at the glass temperature if the latter is far below the crossover. It seems that the dynamic heterogeneity develops only below the crossover. This may help to clarify some controversies regarding dynamic heterogeneity. ${ }^{43,44}$

\section{ACKNOWLEDGMENTS}

We thank the Deutsche Forschungsgemeinschaft DFG (Sonderforschungsbereich 418) and the Fonds der Chemischen Industrie FCI for financial support. Furthermore, we thank Dr. M. Stölzer for preparing the nickel heaters, and Dr. S. Zeeb for sample preparation. 


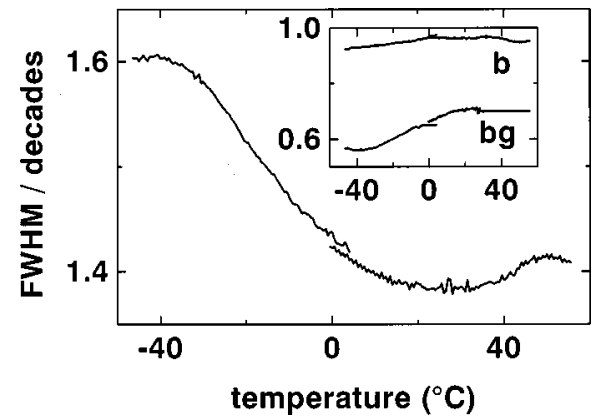

FIG. 11. Temperature dependence of the dielectric full width at the half maximum (FWHM). The inset shows the temperature dependence of the Havriliak-Negami wing parameters $b$ and $b g$.

\section{APPENDIX}

This Appendix is to discuss two further evaluation methods comparing different $\Delta c_{p}(T)$ behavior for the two onset scenarios.

We start with a short remark on the isothermal dielectric FWHM (full width at half $\varepsilon^{\prime \prime}$ maximum). We find a constant value of about 1.40 decades for the $a$ process $\left(T>10^{\circ} \mathrm{C}\right)$ (Fig. 11). For the $\alpha$ process, a sigmoid-shaped increase for lower temperatures is observed, terminating at about $T$ $\approx-30{ }^{\circ} \mathrm{C}$ well above $T_{g}$. The wing analysis of the $\log \varepsilon^{\prime \prime}(\log \omega)$ diagram [Fig. 3(A)] with the aid of HavriliakNegami exponents from Eq. (7) shows that it is the highfrequency wing (product of $b$ and $g$ exponents) that causes the increase of FWHM (inset of Fig. 11).

A set of WLF curves with common asymptotes would result in complementary isothermal ( $\delta \ln \omega \sim$ FWHM) and isochronous $\quad(\delta T) \quad$ widths, $\quad \delta \ln \omega \sim 1 /\left(T-T_{\infty}\right), \quad \delta T \sim(T$ $\left.-T_{\infty}\right), \quad \delta \ln \omega \times \delta T=$ const. $^{8,17,45}$ Consider now the isochronous $\delta T$ dispersions from the $c_{p}^{\prime \prime}(T)$ peaks according to Eq. (2), Table II. They are not complementary to the isothermal dielectric FWHMs. Such a complementariness, however, is not necessary because dielectrics and calorimetry are different activities, and because the traces in the Arrhenius diagram below the crossover are not exact WLF curves [cf. Fig. $7(\mathrm{C})]$. The $\delta T$ dispersion in fact decreases with falling temperatures but the calorimetric $\delta T$ decrease is much larger than the dielectric FWHM increase.

The $\delta T$ decrease supports increasing cooperativities [Eq. (1), Table II]. We shall discuss two variants for a given cooperativity exponent $\nu_{g}=1 / 2$ in Eq. (6).

(vi) In case $\Delta c_{p} \sim\left(T_{\text {on }}-T\right)$, from Eqs. (1) and (5) we would expect

$$
\delta T(T) \cdot\left(T_{\mathrm{on}}-T\right)^{1 / 2}=\text { const. }
$$

(vii) In case $\Delta c_{p}=$ const, from Eqs. (1) and (5) we would expect

$$
\delta T(T) \cdot\left(T-T_{\mathrm{on}}\right)=\text { const. }
$$

The two variants were tested for three random copolymers $\operatorname{poly}(n$-butylmethacrylate-stat-styrene) $P(n \mathrm{BMA}$-stat- $S)$ with 2,8 , and $19 \%$ styrene $e^{25,46,47}\left(T_{\text {on }}=78^{\circ} \mathrm{C}, 83^{\circ} \mathrm{C}\right.$, and $100^{\circ} \mathrm{C}$, respectively). The copolymers belong to the I scenario. Figure 12 shows the product $\delta T\left(T_{\text {on }}-T\right)^{1 / 2}$ (A) and $\delta T\left(T_{\text {on }}\right.$ $-T$ ) (B) vs temperature for the copolymers. To compare the

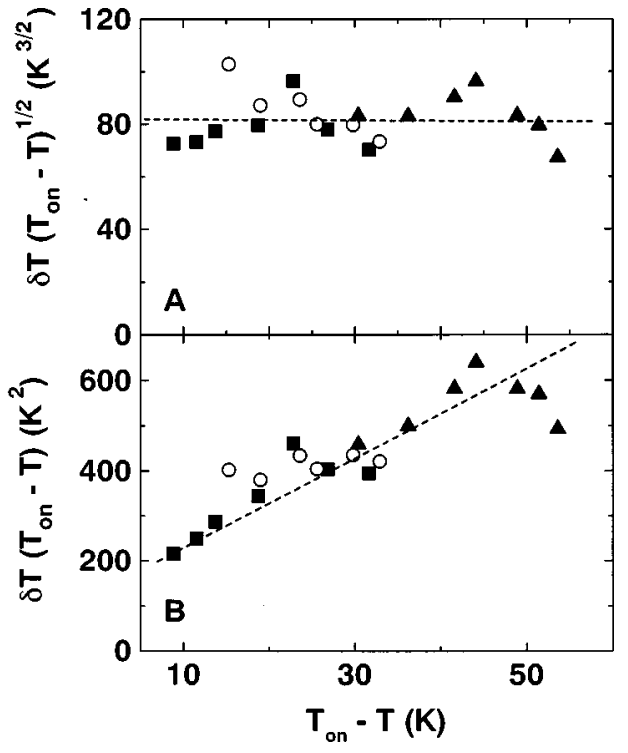

FIG. 12. Product of temperature dispersion and square root of distance to the onset temperature, $x=\left(\delta T\left(T_{\mathrm{on}}-T\right)^{1 / 2}\right)(\mathrm{A})$, and product of dispersion and distance to the onset temperature, $x^{\prime}=\left(\delta T\left(T_{\text {on }}-T\right)\right)(\mathrm{B})$ vs distance to the onset temperature $\left(T_{\text {on }}-T\right)$ for three copolymers poly $(n-$ butylmethacrylate-stat-styrene) with $2(\boldsymbol{\square}), 8(\bigcirc)$, and 19\% styrene $(\boldsymbol{\Lambda})$. The data are from Refs. 25 and 46. The dotted lines are linear fits.

three samples we plot the two products versus the temperature distance from the individual onsets $T_{\text {on }}-T$. We get a uniform picture because the three samples are similar. From Fig. 12(A) we see that the product $\delta T\left(T_{\text {on }}-T\right)^{1 / 2}$ is almost temperature independent, as expected from $\Delta c_{p} \rightarrow 0,{ }^{25,46}$ but the product $\delta T\left(T_{\text {on }}-T\right)$ turns out to depend linearly on tem-
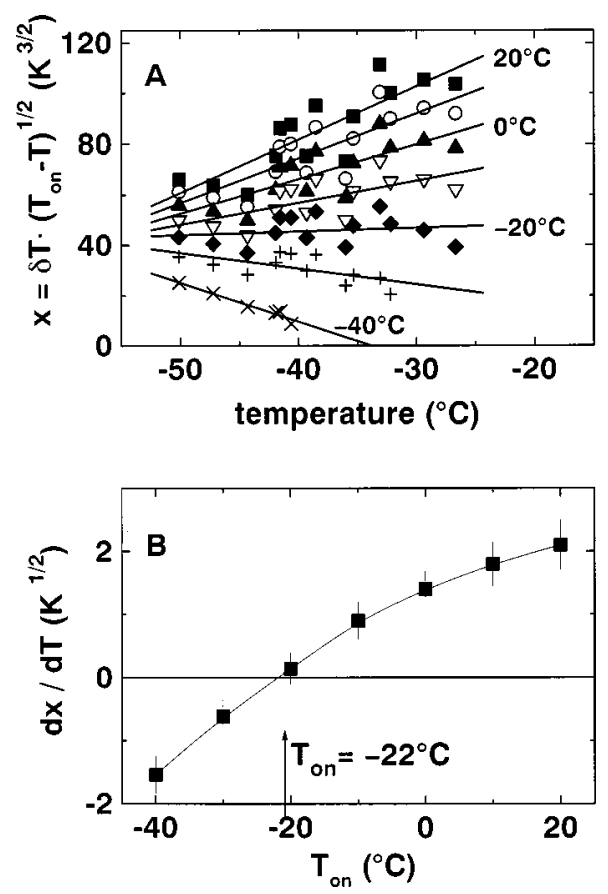

FIG. 13. (A) $x$ product of dispersion and square root of distance to the onset temperature, $x=\delta T\left(T_{\text {on }}-T\right)^{1 / 2}$ vs temperature for BIBE at different hypothetical onset temperatures $T_{\text {on }}\left[T_{\text {on }}=20(\boldsymbol{\square}), 10(\bigcirc), 0(\boldsymbol{\Lambda}),-10(\nabla)\right.$, $-20(\checkmark),-30(+),-40(\times)]$. The lines represent linear fits. (B) The slopes $d / d T\left(\delta T\left(T_{\mathrm{on}}-T\right)^{1 / 2}\right)$ are taken from the linear fits and displayed vs onset temperature $T_{\text {on }}$. The slope is zero at $T_{\text {on }}=-22{ }^{\circ} \mathrm{C} \pm 10 \mathrm{~K}$. 

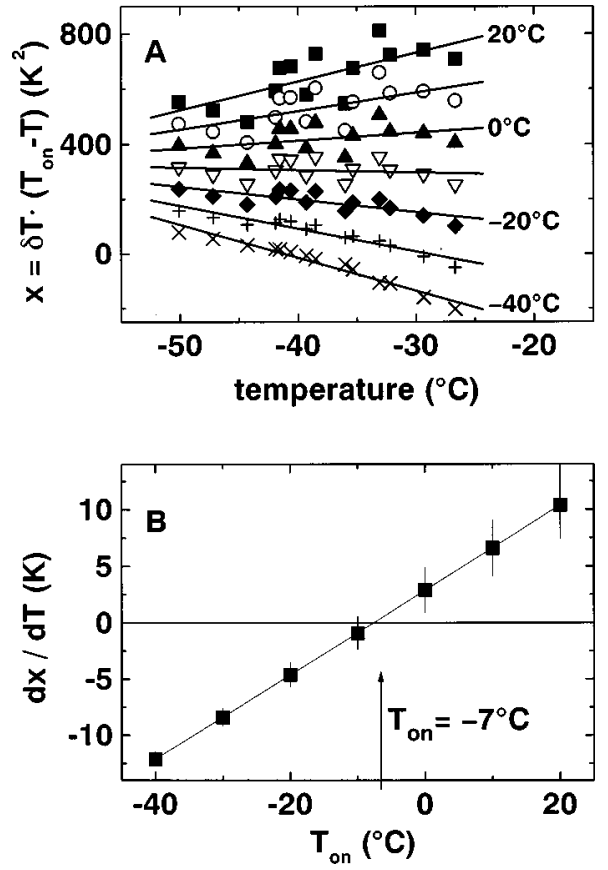

FIG. 14. (A) $x^{\prime}$ product of dispersion and distance to the onset temperature, $\delta T\left(T_{\text {on }}-T\right)$, vs temperature for BIBE at different hypothetical onset temperatures $T_{\text {on }}\left[T_{\text {on }}=20(\boldsymbol{\square}), 10(\bigcirc), 0(\mathbf{\Delta}),-10(\nabla),-20(\downarrow),-30(+)\right.$, $-40(\times)$, same data as for Fig. 13]. The lines represent linear fits. (B) The slopes $d / d T\left(\delta T\left(T_{\text {on }}-T\right)\right)$ are taken from the linear fits and displayed vs onset temperature $T_{\text {on }}$. The slope is zero at $T_{\text {on }}=-7{ }^{\circ} \mathrm{C} \pm 10 \mathrm{~K}$.

perature. The solid lines are linear fits. The variant (vi) is confirmed by the explicit observation of $\Delta c_{p} \sim\left(T_{\mathrm{on}}-T\right)$ according to Eq. (4).

The situation is now analyzed for BIBE. As the $\Delta c_{p}$ values of Table II scatter considerably both variants to estimate the onset temperature were used (Figs. 13 and 14). The slopes for the (vi) variant [Fig. 13(A)] are displayed as a function of the $T_{\text {on }}$ parameter in Fig. 13(B). The onset temperature is obtained from the zero slope as $T_{\text {on }}=-22^{\circ} \mathrm{C}$ $\pm 10 \mathrm{~K}$, corresponding to $\log \omega_{\text {on }}=4.8 \pm 1.0$. The slopes for the (vii) variant (Fig. 14) result in $T_{\text {on }}=-7{ }^{\circ} \mathrm{C} \pm 10 \mathrm{~K}$, corresponding to $\log \omega_{\mathrm{on}}=6.5 \pm 1.0$. Both variants (vi) and (vii) are also incorporated in Fig. 9(B). The (vii) onset temperature agrees well with the $T_{\text {on }}=-8{ }^{\circ} \mathrm{C} \pm 8 \mathrm{~K}$ average of Table III, the (vi) value does not agree. It seems, therefore, that for BIBE the calorimetric intensity $\Delta c_{p}$ in the crossover region is nearly constant [variant (vii)], irrespective of the $N_{\alpha}$ onset.

${ }^{1}$ H. Sillescu, J. Non-Cryst. Solids 243, 81 (1999).

${ }^{2}$ M. D. Ediger, C. A. Angell, and S. R. Nagel, J. Phys. Chem. 100, 13200 (1996).

${ }^{3}$ C. A. Angell, Science 267, 1924 (1995); B. Frick and D. Richter, ibid. 267, 1939 (1995).

${ }^{4}$ C. A. Angell, Curr. Opin. Solid State Mater. Sci. 1, 578 (1996)

${ }^{5}$ W. Götze and L. Sjöjren, Rep. Prog. Phys. 55, 241 (1992).

${ }^{6}$ E. Donth, J. Non-Cryst. Solids 53, 325 (1982).

${ }^{7}$ G. Adam and J. H. Gibbs, J. Chem. Phys. 43, 139 (1965).

${ }^{8}$ E. Donth, Glasübergang (Akademie, Berlin, 1981).

${ }^{9}$ E. Donth, J. Polym. Sci., Part B: Polym. Phys. 34, 2881 (1996).

${ }^{10}$ J. Korus, E. Hempel, M. Beiner, S. Kahle, and E. Donth, Acta Polym. 48, 369 (1997)
${ }^{11}$ E. Hempel, G. Hempel, A. Hensel, C. Schick, and E. Donth, J. Phys. Chem. B. (submitted).

${ }^{12}$ N. G. McCrum, B. E. Read, and G. Williams, Anelastic Effects in Polymeric Solids (Wiley, London, 1967).

${ }^{13}$ G. P. Johari and M. Goldstein, J. Chem. Phys. 53, 2372 (1970).

${ }^{14}$ G. Williams, Trans. Faraday Soc. 62, 2091 (1966).

${ }^{15}$ A. J. Barlow, J. Lamb, and A. J. Matheson, Proc. R. Soc. London, Ser. A 292, 322 (1966).

${ }^{16}$ M. Cukierman, J. W. Lane, and D. R. Uhlmann, J. Chem. Phys. 59, 3639 (1973); W. T. Laughlin and D. R. Uhlmann, J. Phys. Chem. 76, 2317 (1972)

${ }^{17}$ E. Donth, Relaxation and Thermodynamics in Polymers. Glass Transition (Akademie, Berlin, 1992).

${ }^{18}$ F. Stickel, E. W. Fischer, and R. Richert, J. Chem. Phys. 102, 6251 (1995); 104, 2043 (1996); F. Stickel, thesis, Universität Mainz, Shaker, 1995.

${ }^{19}$ M. L. Williams, R. F. Landel, and J. D. Ferry, J. Am. Chem. Soc. 77, 3701 (1955).

${ }^{20}$ E. Rössler, Phys. Rev. Lett. 65, 1595 (1990).

${ }^{21}$ F. Fujara, B. Geil, H. Sillescu, and G. Fleischer, Z. Phys. B 88, 195 (1992).

${ }^{22}$ A. P. Sokolov, Science 273, 1675 (1996); Endeavour 21, 109 (1997).

${ }^{23}$ M. Beiner, S. Kahle, E. Hempel, K. Schröter, and E. Donth, Europhys. Lett. 44, 323 (1998)

${ }^{24}$ F. Garwe, A. Schönhals, H. Lockwenz, M. Beiner, K. Schröter, and E. Donth, Macromolecules 29, 247 (1996).

${ }^{25}$ S. Kahle, J. Korus, E. Hempel, R. Unger, S. Höring, K. Schröter, and E. Donth, Macromolecules 30, 7214 (1997).

${ }^{26}$ A. Arbe, D. Richter, J. Colmenero, and B. Farago, Phys. Rev. E 54, 3853 (1996)

${ }^{27}$ R. Bergman, F. Alvarez, A. Alegria, and J. Colmenero, J. Chem. Phys. 109, 7546 (1998).

${ }^{28}$ D. Fioretto, L. Comez, G. Socino, L. Verdini, S. Corezzi, and P. A. Rolla, Phys. Rev. E 59, 1899 (1999)

${ }^{29}$ C. Hansen, F. Stickel, T. Berger, R. Richert, and E. W. Fischer, J. Chem. Phys. 108, 6408 (1998).

${ }^{30}$ A. Schönhals and F. Kremer, J. Non-Cryst. Solids 172-174, 336 (1994).

${ }^{31}$ A. Kudlik, C. Tschirwitzs, S. Benkhof, T. Blochowicz, and E. Rössler, Europhys. Lett. 40, 649 (1997).

${ }^{32}$ J. Korus, M. Beiner, K. Busse, S. Kahle, R. Unger, and E. Donth, Thermochim. Acta 304/305, 99 (1997).

${ }^{33}$ W. H. Press, P. B. Flannery, S. A. Teukolsky, and W. T. Vetterling, Numerical Recipes in Pascal (Fortran, C). The Art of Scientific Computing (Cambridge Univ. Press, Cambridge and New York, 1992).

${ }^{34}$ S. Havriliak and S. Negami, J. Polym. Sci., Part C: Polym. Symp. 14, 99 (1966).

${ }^{35}$ N. F. Mott and R. A. Davis, Electronic Processes in Non-Crystalline Materials (Clarendon, Oxford, England, 1979).

${ }^{36}$ F. Alvarez, A. Hofman, A. Alegria, and J. Colmenero, J. Chem. Phys. 105, 432 (1996).

${ }^{37}$ C. F. Böttcher and P. Bordewijk, Theory of Electric Polarization, Vol.2: Dielectrics in Time Dependent Fields (Elsevier, Amsterdam, 1978).

${ }^{38}$ N. O. Birge, Phys. Rev. B 34, 1631 (1986); N. O. Birge and S. R. Nagel, Rev. Sci. Instrum. 58, 1464 (1987).

${ }^{39}$ N. O. Birge and N. Menon, Thermochim Acta 305, 51 (1997).

${ }^{40}$ C. T. Moynihan, A. J. Easteal, J. Wilder, and J. Tucker, J. Phys. Chem. 78, 2673 (1974)

${ }^{41}$ H. Vogel, Phys. Z. 22, 645 (1921); G. S. Fulcher, J. Am. Chem. Soc. 8, 339 (1925); G. Tammann and G. Hesse, Z. Anorg. Allg. Chem. 156, 245 (1926)

${ }^{42}$ See, e.g., N. W. Tschoegl, The Phenomenological Theory of Linear Viscoelastic Behavior. An Introduction (Springer, Berlin, 1989).

${ }^{43}$ A. Heuer and H. W. Spiess, Phys. Rev. Lett. 82, 1335 (1999).

${ }^{44}$ A. Arbe, J. Colmenero, M. Monkenbusch, and D. Richter, Phys. Rev. Lett. 82, 1336 (1999).

${ }^{45}$ E. Donth, J. Phys. I 6, 1189 (1996).

${ }^{46}$ S. Kahle, thesis, Universität Halle, 1997.

${ }^{47}$ J. Korus, thesis, Universität Halle, 1997. 